\title{
ORDER RELATION IN JORDAN RINGS AND A STRUCTURE THEOREM
}

\author{
SANTOS GONZÁLEZ AND CONSUELO MARTÍNEZ ${ }^{1}$
}

\begin{abstract}
It is shown that the relation $\leqslant$ defined by $x \leqslant y$ if and only if $x y=x^{2}$, $x^{2} y=x y^{2}=x^{3}$ is an order relation for a class of Jordan rings and we prove that a Jordan ring $R$ is isomorphic to a direct product of Jordan division rings if and only if $\leqslant$ is a partial order on $R$ such that $R$ is hyperatomic and orthogonally complete.
\end{abstract}

Introduction. The usual relation in Boolean rings is extended to reduced rings $A$ (has no nilpotent elements) when it is expressed as $a \leqslant b$ if and only if $a b=a^{2}$ (Abian [1, 2] and Chacron [4]) and it is proved that any ring $R$ equipped with the relation $\leqslant$ is isomorphic to a direct product of division rings if and only if $\leqslant$ is an order relation in $R$ such that $R$ is hyperatomic and orthogonally complete. In [5] Myung and Jiménez extend the above results to any alternative ring and they show that the same results do not hold for Jordan rings, because the ring $Q$ of real quaternions under the product $a \cdot b=\frac{1}{2}(a b+b a)$ becomes a Jordan ring $Q^{+}$ without nonzero nilpotent elements, but the relation $\leqslant$ is not a partial order on $Q^{+}$. Also, $Q^{+}$is a Jordan division ring, in the sense that $U_{a}=2 R_{a}^{2}-R_{a^{2}}$ is invertible on $Q^{+}$for every $a \neq 0$ in $Q^{+}$, where $R_{a}$ is the right multiplication in $Q^{+}$ by $a$.

The aim of this paper is to define an order relation for Jordan rings and to obtain a structure theorem for these rings.

1. Preliminaries. In this section we give the elementary properties and definitions related to Jordan rings which will be needed in the paper.

A Jordan ring is a commutative nonassociative ring $R$ satisfying $(x y) x^{2}=x\left(y x^{2}\right)$ for all $x, y \in R$. In terms of the associator $(x, y, z)=(x y) z-x(y z)$ this is to say $\left(x, y, x^{2}\right)=0$. If $A$ is an associative ring then it is well known that the elements of $A$ form a Jordan ring under the same operation of addition and under the new multiplication $a \cdot b=\frac{1}{2}(a b+b a)$ where $a b$ denote the associative product of $a$ and $b$ in $A$. This ring will be denoted by $A^{+}$. It is easy to see that the powers of an element in $A$ are the same under the Jordan product as under the associative product, more generally any subring of a Jordan ring which is generated by one

Received by the editors June 11, 1985 and, in revised form, October 28, 1985.

1980 Mathematics Subject Classification (1985 Revision). Primary 17C10.

Key words and phrases. Jordan ring, partial order, direct product, nonzero nilpotent element, hyperatom, orthogonally complete.

${ }^{1}$ Supported by C. A. Investigación Científica y Técnica, n. 0778-84. 
element is associative, and then the concept of a nilpotent element carries over from the associative theory to Jordan theory without ambiguity.

In Jordan rings we shall need to use the operator $U_{x}$ defined for each $x \in R$ by $y U_{x}=2(y x) x-y x^{2}$ for all $y \in R$. If $1 \in R$, we say that an element $a \in R$ has $b$ as an inverse if $a b=1, a^{2} b=a$ and it can be shown that $a \in R$ is invertible if and only if the operator $U_{a}$ is invertible on $R$.

The identities used most in the paper are the following:

$$
\begin{aligned}
& x^{n} y=2 x^{n-1}(x y)-2 x^{n-2}(x y x)+x^{n-2}\left(x^{2} y\right) \quad \forall x, y \in R, \forall n \geqslant 2 \\
& x^{n}\left(y x^{m}\right)=\left(x^{n} y\right) x^{m} \quad \forall x, y \in R, \forall n, m \geqslant 0 \\
& \begin{aligned}
((x y) z) u+((x u) z) y+((y u) z) x & =(x y)(z u)+(x z)(y u)+(x u)(y z) \\
& =((x y) u) z+((x z) u) y+((y z) u) x
\end{aligned}
\end{aligned}
$$

That is to say, using the associators

$$
\begin{aligned}
& (x y, z, u)+(x u, z, y)+(y u, z, x)=0, \\
& (z, x y, u)=(z, x, u) y+(z, y, u) x,
\end{aligned} \quad \forall x, y, z, u \in R .
$$

(3) By the commutativity of $R$, we have

$$
\begin{aligned}
& (x, y, x)=0, \text { or equivalently }(x, y, z)=-(z, y, x), \quad \forall x, y, z \in R \\
& (x, y, z)+(y, z, x)+(z, x, y)=0,
\end{aligned}
$$

2. Order relation. Let $R$ be a Jordan ring in which $2 x=0$ implies $x=0$ for all $x \in R$. We define the relation

$$
x \leqslant y \quad \text { iff } \quad x y=x^{2}, \quad x^{2} y=x y^{2}=x^{3} .
$$

If $x \leqslant y$, then $(x, x, y)=(x, y, y)=0$. Thus, using the identities (1), $x^{i} y=x^{i+1}$, $x^{i} y^{j}=x^{i+j}$ for all $i, j \geqslant 1$, and the algebra generated by $x$ and $y$ is an associative algebra.

Let us observe immediately that if $\leqslant$ is a partial order on $R$, then $R$ has no nilpotent elements $(\neq 0)$ because $x^{2}=0$ implies $x \leqslant x^{2}$ and $x^{2} \leqslant x$, hence $x=x^{2}$ $=0$. In what follows $R$ always stands for a Jordan ring without nonzero nilpotent elements.

LEMMA 1. The relation $\leqslant$ is reflexive and antisymmetric.

Proof. Clearly $\leqslant$ is reflexive. If $x \leqslant y$ and $y \leqslant x$ then $x y=x^{2}, y x=y^{2}$. So $x^{2}-x y-y x+y^{2}=(x-y)^{2}=0$. But then $x-y=0$ and so $x=y$.

Now let $R$ be a Jordan ring without nonzero nilpotent elements satisfying the property $(\mathrm{P})$ given by $(x, x, y)=0$ implies $(x y, x, y)=0$.

There exist Jordan rings without nonzero nilpotent elements which are not associative and satisfying (P). For example the real quaternions $Q^{+}$.

THEOREM 1. Let $R$ be a Jordan ring without nonzero nilpotent elements and satisfying (P). Then the relation $\leqslant$ is a partial order on $R$. 
Proof. Let $x \leqslant y$ and $y \leqslant z$. Then $x y=x^{2}, x^{2} y=x y^{2}=x^{3}$ and $y z=y^{2}$, $y^{2} z=y z^{2}=y^{3}$. Then

(i) $\left(x^{2}, x, z\right)+2(x z, x, x)=0$. This implies $3 x z x^{2}=x^{3} z+2 x(x z x)$.

(ii) $\left(x^{2}, z, y\right)+2(x y, z, x)=0$ and since $(x y, z, x)=0=\left(x^{2}, z, x\right)$, we have $\left(x^{2}, z, y\right)=\left(x^{2} z\right) y-x^{2}(z y)=0$.

(iii) $(x y, z, y)+\left(y^{2}, z, x\right)+(x y, z, y)=0$. Then, using (ii), we get $\left(y^{2}, z, x\right)=0$, that is $y^{2}(z x)=\left(y^{2} z\right) x=y^{3} x=x^{4}$.

(iv) $\left(y^{2}, x, z\right)+2(y z, x, y)=0=\left(y^{2}, x, z\right)+2\left(y^{2}, x, y\right)$. Then $\left(y^{2}, x, z\right)=0$, that is $\left(y^{2} x\right) z=y^{2}(x z)=x^{3} z$.

(v) $x^{3} z=y^{2}(x z)=y^{3} x=x^{4}=\left(x^{2} z\right) y$ by (iii) and (iv).

(vi) $(x y, y, z)+(x z, y, y)+(y z, y, x)=0$ so $\left(x^{2}, y, z\right)+(x z, y, y)+\left(y^{2}, y, x\right)$ $=0$. Since $\left(y^{2}, y, x\right)=0$, we have $\left(x^{2} y\right) z-x^{2}(y z)+((x z) y) y-(x z) y^{2}=0$ and then $\left(x^{2} y\right) z=x^{3} z=x^{4}=x^{2}(y z)=x^{2} y^{2}$ by (v). Thus $((x z) y) y=(x z) y^{2}=x^{4}$.

(vii) $x^{4}=x^{3} z=y^{3} x=y^{2}(x z)=\left(x^{2} z\right) y=((x z) y) y$ using (v) and (vi).

(viii) $\left(x^{2}, y, z\right)+2(x z, y, x)=0$, and since $\left(x^{2}, y, z\right)=\left(x^{2} y\right) z-x^{2}(y z)=x^{3} z$ $-x^{2} y^{2}=x^{3} z-x^{4}=0$, we get $(x z, y, x)=0$, that is $((x z) y) x=(x z)(y x)=$ $(x z) x^{2}$.

(ix) As $x^{3} z=x^{4}$, we have $\left(x^{3}, z, y\right)=0=\left(x^{2} y, z, y\right)$. But $2\left(x^{2} y, z, y\right)+$ $\left(y^{2}, z, x^{2}\right)=0$ implies with the above that $\left(y^{2}, z, x^{2}\right)=0$ and so $\left(y^{2} z\right) x^{2}=$ $y^{2}\left(z x^{2}\right)=y^{3} x^{2}=x^{5}$. On the other hand, $\left(x^{3}, y, z\right)+\left(y, z, x^{3}\right)+\left(z, x^{3}, y\right)=0$ and $\left(x^{3}, z, y\right)=-\left(y, z, x^{3}\right)=0$ imply that

$$
\begin{aligned}
\left(x^{3}, y, z\right) & =-\left(z, x^{3}, y\right)=-\left(z, x y^{2}, y\right) \\
& =-(z, x, y) y^{2}-\left(z, y^{2}, y\right) x=-y^{2}\left((z x) y-z x^{2}\right) .
\end{aligned}
$$

But $y^{2}(z x) y=\left(y^{2}(z x)\right) y=x^{4} y=x^{5}$ by (vii), hence $\left(x^{3}, y, z\right)=0$ implies $x^{4} z=$ $x^{3}(y z)=x^{3} y^{2}=x^{5}$.

(x) Using the identities (1), $x^{n} z=x^{n+1} \forall n \geqslant 4$.

(xi) $\left(x^{2} z x\right) x^{2}=\left(x^{2} z\right) x^{3}+\left(x^{2} z, x, x^{2}\right)=x^{2}\left(z x^{3}\right)=x^{6}$, since $2\left(x^{2} z, x, x^{2}\right)+$ $\left(x^{4}, x, z\right)=0$ and, by $(\mathrm{x}),\left(x^{4}, x, z\right)=0$.

(xii) $\left(x^{2} z x\right)^{2}=x^{8}$. In fact,

$$
\left(x^{2} z x\right)^{2}=\left(x^{2} z x\right)\left(x^{2}(z x)\right)=\left(\left(x^{2} z x\right) x^{2}\right)(z x)-\left(x^{2} z x, x^{2}, z x\right) .
$$

But $\left(x^{2}, x^{2}, z x\right)=x^{4} z x-x^{2}\left(x^{2} z x\right)=x^{6}-x^{6}=0$ by (xi). Then property (P) implies $\left(x^{2} z x, x^{2}, z x\right)=0$. Hence we get $\left(x^{2} z x\right)^{2}=\left(\left(x^{2} z x\right) x^{2}\right)(z x)=x^{6} z x=x^{8}$ using (x).

(xiii) $x^{4}=x^{2} z x$. In fact,

$$
\left(x^{2} z x-x^{4}\right)^{2}=\left(x^{2} z x\right)^{2}-2\left(x^{2} z x\right) x^{4}+x^{8}=2 x^{8}-2 x^{8}=0 .
$$

Hence $x^{2} z x=x^{4}$ that is to say $\left(x^{2}, x, z\right)=0$.

(xiv) $\left(x^{2}, x, z\right)+2(x z, x, x)=0$ and (xiii) implies $(x z, x, x)=0$ and so $(x z x) x=$ $x z x^{2}=x^{4}=\left(x^{2} z\right) y$ by (ii).

(xv) $x^{2} z x^{2}=x^{5}$. In fact,

$$
x^{4} z=x^{5}=2 x^{3}(x z)-2 x^{2}(s x z x)+x^{2}\left(z x^{2}\right)=2 x^{5}-2 x^{5}+x^{2} z x^{2}=x^{2} z x^{2} \text {. }
$$


(xvi) $x^{2} z=x^{3}$. In fact,

$$
\begin{aligned}
\left(x^{2} z-x^{3}\right)^{2} & =\left(x^{2} z\right)\left(x^{2} z\right)-2\left(x^{2} z\right) x^{3}+x^{6}=\left(x^{2} z\right)\left(x^{2} z\right)-2\left(x^{3} z\right) x^{2}+x^{6} \\
& =\left(x^{2} z x^{2}\right) z-\left(x^{2} z, x^{2}, z\right)-x^{6}=x^{5} z-x^{6}-\left(x^{2} z, x^{2}, z\right)=0
\end{aligned}
$$

by (xv), because $x^{5} z=x^{6}$ and $\left(x^{2}, x^{2}, z\right)=x^{4} z-x^{2} z x^{2}=x^{5}-x^{5}=0$ implies $\left(x^{2} z, x^{2}, z\right)=0$ by (P). Hence we obtain $x^{2} z=x^{3}$.

(xvii) $x z x=x^{3}$. In fact,

$$
\left(x z x-x^{3}\right)^{2}=(x z x)^{2}-2 x^{3}(x z x)+x^{6}=(x z x)^{2}-x^{6}=0
$$

because

$$
(x z x)^{2}=(x z x)(x z x)=((x z x) x)(z x)-(x z x, x, z x)=x^{4} z x=x^{6}
$$

using (xiv) and that $(x, x, z x)=-(z x, x, x)=0$ implies $(x z x, x, z x)=0$ by property (P).

(xviii) $x z=x^{2}$. In fact,

$$
\left(x z-x^{2}\right)^{2}=(x z)^{2}-2 x^{2}(x z)+x^{4}=(x z)^{2}-x^{4}=0
$$

because

$$
(x z)^{2}=(x z)(x z)=(x z x) z-(x z, x, z)=x^{3} z=x^{4}
$$

because $(x, x, z)=x^{2} z-x z x=x^{3}-x^{3}=0$ (by (xvi) and (xvii)) and property (P) implies $(x z, x, z)=0$. Finally

(xix) $x z^{2}=x^{3}$. In fact, $x \leqslant y$ and $y \leqslant z$ implies $x^{2} \leqslant y^{2}$ and $y^{2} \leqslant z^{2}$. By the above results for $x^{2}, y^{2}, z^{2}$ we have $x^{2} z^{2}=\left(x^{2}\right)^{2}=x^{4}$ (xviii), and $x^{4} z^{2}=x^{6}$ (xvi). Also $\left(x, x, z^{2}\right)+2(z, x, x z)=0$ and $(x z, x, z)=-(z, x, z x)=0$ by (xviii), then $\left(x, x, z^{2}\right)=0$. Hence, $x z^{2} x=x\left(x z^{2}\right)=x^{2} z^{2}-\left(x, x, z^{2}\right)=x^{2} z^{2}=x^{4}$ and so

$$
\left(x z^{2}\right)^{2}=\left(x z^{2}\right)\left(x z^{2}\right)=\left(x z^{2} x\right) z^{2}-\left(x z^{2}, x, z^{2}\right)=x^{4} z^{2}=x^{6}
$$

because $\left(x, x, z^{2}\right)=0$ implies $\left(x z^{2}, x, z^{2}\right)=0$ by property $(\mathrm{P})$.

Thus,

$$
\left(x z^{2}-x^{3}\right)^{2}=\left(x z^{2}\right)^{2}-2 x^{3}\left(x z^{2}\right)+x^{6}=2 x^{6}-2 x^{3}\left(x z^{2}\right)=0,
$$

because $\left(x, x, z^{2}\right)=0$ implies $2 x\left(x, x, z^{2}\right)=\left(x, x^{2}, z^{2}\right)=0$ and so $x\left(x^{2} z^{2}\right)=x^{3} z^{2}$, that is $x^{3}\left(z^{2} x\right)=\left(x^{3} z^{2}\right) x=x^{5} x=x^{6}$. Hence $x z^{2}=x^{3}$. Then $\leqslant$ is a partial order on $R$ and the theorem is proved.

THEOREM 2. Let $R$ be a special Jordan ring whose special universal envelope is an associative algebra without nilpotent elements. Then $\leqslant$ is a partial order on $R$.

Proof. Let $\cdot$ be the associative product on $R$ such that $x y=\frac{1}{2}(x \cdot y+y \cdot x)$. By $\leqslant$ we denote the Abian's order relation on the envelope associative and we will prove that $a \leqslant b$ if and only if $a \leqslant b$.

If $a \leqslant b$ we have $a b=a^{2}$ and $a^{2} b=a b^{2}=a^{3}$, that is $(a, a, b)=0=(b, b, a)$. Now, $a^{2} b=a(a b)$ implies $\frac{1}{2}\left(a^{2} \cdot b+b \cdot a^{2}\right)=\frac{1}{4}(a \cdot a \cdot b+a \cdot b \cdot a+a \cdot b \cdot a+$ $b \cdot a \cdot a)$ and so

$$
a^{2} \cdot b+b \cdot a^{2}=a \cdot a \cdot b+b \cdot a \cdot a=2 a \cdot b \cdot a .
$$


Similarly $(b, b, a)=0$ implies

$$
b \cdot b \cdot a+a \cdot b \cdot b=2 b \cdot a \cdot b .
$$

With $b \cdot(*)$ we have $b \cdot a^{2} \cdot b+b^{2} \cdot a^{2}=2 b \cdot a \cdot b \cdot a$ and $(* *) \cdot a$ implies $b^{2} \cdot a^{2}+a \cdot b^{2} \cdot a=2 b \cdot a \cdot b \cdot a$. Hence $b \cdot a^{2} \cdot b=a \cdot b^{2} \cdot a$.

Also $a^{2}=a \cdot a=a b=\frac{1}{2}(a \cdot b+b \cdot a)$, therefore $(a \cdot b+b \cdot a) \cdot a=a \cdot(a \cdot b$ $+b \cdot a)$. Using the abovementioned and (*), we get $a \cdot b \cdot a=a^{2} \cdot b=b \cdot a^{2}$. Hence

$$
b \cdot a^{2} \cdot b=b \cdot\left(a^{2} \cdot b\right)=b \cdot b \cdot a^{2}=b^{2} \cdot a^{2}=b \cdot(a \cdot b \cdot a)=b \cdot a \cdot b \cdot a .
$$

So

$$
b \cdot a^{2} \cdot b=a^{2} \cdot b \cdot b=a^{2} \cdot b^{2}=a \cdot b \cdot a \cdot b
$$

and

$$
a \cdot b^{2} \cdot a=2 a \cdot b \cdot a \cdot b-a^{2} \cdot b^{2}=a \cdot b \cdot a \cdot b=a^{2} \cdot b^{2}=b^{2} \cdot a^{2}=b \cdot a^{2} \cdot b .
$$

Then

$$
(a \cdot b-b \cdot a)^{2}=a \cdot b \cdot a \cdot b+b \cdot a \cdot b \cdot a-a \cdot b \cdot b \cdot a-b \cdot a \cdot a \cdot b=0 .
$$

So $a \cdot b=b \cdot a=a b$ and $a \cdot b=a^{2}$, that is $a \leqslant b$.

Conversely, let $a \leqslant b$. We see that $a \cdot b=b \cdot a$ because

$$
\begin{aligned}
\left(b \cdot a-a^{2}\right)^{2} & =b \cdot a \cdot b \cdot a-b \cdot a \cdot a^{2}-a^{2} \cdot b \cdot a+a^{4} \\
& =b \cdot a^{3}-b \cdot a^{3}-a^{4}+a^{4}=0
\end{aligned}
$$

and so

$$
\begin{gathered}
a \cdot b=a b=a^{2}, \\
a^{2} b=a^{2} \cdot b=a \cdot a \cdot b=a^{3}=b^{2} a=b \cdot b \cdot a=b \cdot a^{2} .
\end{gathered}
$$

Hence $a \leqslant b$.

Consequently $\leqslant$ is a partial order on $R$ because $\leqslant$ is a partial order on the associative envelope by Abian's result.

REMARK. It is known that $a \leqslant b$ is an associative ring implies $a b=b a$. The same result is true for an alternative ring.

Question. Is the relation that we have defined above an order relation on every Jordan ring without nilpotent elements?

3. A structure theorem. Let $R$ be a Jordan ring and $e$ an idempotent element of $R$. Then $R=R_{0} \dot{+} R_{1 / 2}+R_{1}$ with $R_{0}=\{x \in R \mid x e=0\}, \quad R_{1 / 2}=\{x \in R \mid x e$ $\left.=\frac{1}{2} x\right\}, R_{1}=\{x \in R \mid x e=x\}$ (Peirce's decomposition).

LemMA 1. Let $R$ be a Jordan ring, $R \subseteq S^{+}$, where $S$ is an associative ring without nonzero nilpotent elements and let $e$ be an idempotent element in $R$. Then $(x e) e=x e$ for every $x$ in $R$.

Proof. Let - be the associative product in $S$, and denote by juxtaposition the product in $R$. Since $S$ has no nonzero nilpotent elements, $e$ (and every idempotent element of $S$ ) commutes with every element of $S$, and so $e \cdot x=x \cdot e=x e$, and $(x e) e=(x \cdot e) \cdot e=x \cdot e \cdot e=x \cdot e=x e$. 
LEMMA 2. Let $R$ be a Jordan ring and let e be an idempotent element in $R$ such that $(x, e, e)=0$ for every $x$ in $R$. Then

(i) $R_{1 / 2}(e)=0$.

(ii) $(x, e, y)=0$ for every $x, y$ in $R$.

(iii) $(x, y, e)=(e, y, x)=0$ for every $x, y$ in $R$.

(iv) $R e$ is an ideal of $R$ and $e$ is the unit element of $R e$.

Proof. (i) If $x \in R_{1 / 2}(e), x e=\frac{1}{2} x$ and so $\frac{1}{2} x=x e=(x e) e=\left(\frac{1}{2} x\right) e$ $=\frac{1}{2} x e=\frac{1}{4} x$. Thus $x=0$.

(ii) $(x, e, y)=\left(x, e^{2}, y\right)=2 e(x, e, y)$. Then $(x, e, y) \in R_{1 / 2}(e)$. By (i), $(x, e, y)$ $=0$.

(iii) As $(x, y, e)=-(e, y, x)$, it suffices to show that $(e, y, x)=0$. From $(e, y, x)$ $=\left(e^{2}, y, x\right)$ and $\left(e^{2}, y, x\right)+2(e x, y, e)=0$, we have $\left(e^{2}, y, x\right)=-2(e x, y, e)$. Also

$$
(e x, y, e)+(y, e, e x)+(e, e x, y)=0=(e x, y, e)+(e, e x, y)
$$

by (ii). Therefore

$$
\begin{aligned}
(e, y, x) & =\left(e^{2}, y, x\right)=-2(e x, y, e)=2(e, e x, y) \\
& =2((e, x, y) e+(e, e, y) x)=2(e, x, y) e
\end{aligned}
$$

But

$$
(e, x, y)+(x, y, e)+(y, e, x)=0=(e, x, y)+(x, y, e),
$$

and so $(e, x, y)=-(x, y, e)=(e, y, x)$. We have finally obtained that $e(e, y, x)$ $=\frac{1}{2}(e, y, x)$, that is $(e, y, x) \in R_{1 / 2}(e)$. Therefore $(e, y, x)=0$.

(iv) Let $x e \in R e, y \in R$. Then $y(x e)=(y x) e \in R e$ by (iii). Since $e e=e^{2}=e \in$ $R e$ and $(x e) e=x e$, the element $e$ is the unit element of $R e$.

Definition. Let $R$ be a Jordan ring. An idempotent element $e \neq 0$ of $R$ is called a hyperatom in $R$ if, for every $x$ in $R$

(i) $x \leqslant e$ implies $x=0$ or $x=e$,

(ii) $x e \neq 0$ implies that there exists an element $y$ in $R$ such that (xe) $y=0$ and $(x, x, y)=e$, and

(iii) $(x, e, e)=0$.

REMARK 1. $(x, x, y)=0$ implies $(x e, x e, y)=0$ for a hyperatom $e$, because

$$
\begin{aligned}
(x e, x e, y) & =x(x e, e, y)+e(x e, x, y)=e(x e, x, y)=(x e, x, y) \\
& =(x e x) y-(x e)(x y)=\left(e x^{2}\right) y-e(x y x)=e\left(x^{2} y-x y x\right) \\
& =e(x, x, y)=0 .
\end{aligned}
$$

REMARK 2. If $R$ is a Jordan division ring it is easy to see that 1 is the only idempotent element in $R$ and it is a hyperatom (it is clear that $\leqslant$ is a partial order in $R$ ).

DEFINITION. Let $R$ be a Jordan ring. $R$ is called hyperatomic if for every nonzero element in $R$, there exists a hyperatom $e$ in $R$ such that $e x \neq 0$.

If $R$ is an associative commutative ring, it is easy to see that the definition above and Abian's definition are equivalent (see [1]). 
Proposition 1. Let $A$ be an associative ring without nonzero nilpotent elements. If $A$ is hyperatomic (see [ 2 or 4] for the definition) then $A^{+}$is hyperatomic.

Proof. By [4, Lemma 5], for every nonzero element $x$ in $A$ there exists an idempotent hyperatom $e$ such that $e \cdot x \neq 0$. Also $e$ is in the center of $A$, and so $e$ is an idempotent element of $A^{+}$such that $e \cdot x=e x \neq 0$. (We use the same notation as in Theorem 2 and - denotes the associative product.) Therefore $e$ satisfies the conditions (i) and (iii) of the definition of hyperatom, usirg Lemma 1. Also $e \cdot a \neq 0$ if and only if $e a \neq 0$, and in such case, there exists an element $b$ such that $(a \cdot e) \cdot b=e$. Since $a \cdot e=a e$ and $A e$ is a division ring, $a$ and $b e$ commute and $b$ and $a e$ commute. If $b^{\prime}=b e$, it is clear that $(a e) b^{\prime}=(a \cdot e) \cdot b^{\prime}=(a \cdot e) \cdot b=e$ and $\left(a, a, b^{\prime}\right)=0$.

Hence $A^{+}$is hyperatomic, as desired.

Proposition 2. If e is a hyperatom in $R$, then Re is a Jordan division ring.

Proof. For every $x e \neq 0$ in $\operatorname{Re}$, there exists an element $y$ such that $(x e) y=e$ and $(x, x, y)=0$. Then $(x e)(y e)=(x e y) e=e e=e$. Let $z=y e$. Thus

$$
(x e)^{2} z=((x e)(x e)) z=(((x e)(x e)) y) e=((x e)(x e y)) e=(x e e) e=x e
$$

using Remark 1 and Lemma 2. Therefore $x e$ is an invertible element in $R$ and $z$ is its inverse element, and so $\mathrm{Re}$ is a Jordan division ring, as desired.

LemMA 3. Let $e_{i}, e_{j}$ be two hyperatoms of $R$. Then $e_{i} e_{j}=0$ and $R e_{i} \cap R e_{j}=0$.

Proof. Since $\left(e_{i} e_{j}\right) e_{i}=e_{j} e_{i}=\left(e_{i} e_{j}\right) e_{j}$ we have

$$
\left(e_{i} e_{j}\right)^{2}=\left(e_{i} e_{j}\right)\left(e_{i} e_{j}\right)=\left(e_{i} e_{j}\right) e_{i}=\left(e_{i} e_{j}\right) e_{j}=e_{i} e_{j}
$$

Similarly $\left(e_{i} e_{j}\right)^{2} e_{i}=\left(e_{i} e_{j}\right) e_{i}^{2}=e_{i} e_{j}=\left(e_{i} e_{j}\right)^{3}$ and $\left(e_{i} e_{j}\right)^{2} e_{j}=\left(e_{i} e_{j}\right)^{3}$. Hence $e_{i} e_{j} \leqslant$ $e_{i}, e_{i} e_{j} \leqslant e_{j}$ and so $e_{i} e_{j}=0$. Finally, if $x \in R e_{i} \cap R e_{j}$ we have $x=x e_{i}=x e_{j}$, then $x=\left(x e_{i}\right) e_{j}=x\left(e_{i} e_{j}\right)=0$.

Lemma 4. Let $R$ be hyperatomic and $\left(e_{i}\right)_{i \in I}$ be the set of all hyperatoms of $R$. Then

(i) For every $r \in R, \sup _{i}\left(r e_{i}\right)$ exists and $r=\sup _{i}\left(r e_{i}\right)$.

(ii) The function $\alpha$ defined by $\alpha(x)=\left(x e_{i}\right)_{i \in I}$ is a monomorphism from $R$ into a direct product of Jordan division rings $R_{i}$.

Proof. (i) If $r \neq 0$, it is clear that there exists $e_{j}$ such that $r e_{j} \neq 0$. Now, $\left(r e_{i}\right) r=r^{2} e_{i}=r^{2} e_{i}^{2}=\left(r e_{i}\right)^{2},\left(r e_{i}\right) r^{2}=r^{3} e_{i}=r^{3} e_{i}^{3}=\left(r e_{i}\right)^{3}$ and so $r e_{i} \leqslant r$ for every $i$, that is $r$ is an upper bound of the set $\left(r e_{i}\right)_{i \in I}$. Let $u$ be any upper bound of $\left(r e_{i}\right)_{i \in I}$. Then $\left(r e_{i}\right) u=\left(r e_{i}\right)^{2}=r^{2} e_{i},\left(r e_{i}\right)^{2} u=r^{2} e_{i} u=r e_{i} u^{2}=\left(r e_{i}\right)^{3}=r^{3} e_{i}$ for every $i$. Then $u r=r^{2}$. Assume, on the contrary, that $u r-r^{2} \neq 0$. Thus there exists $e_{i}$ such that $0 \neq(u r) e_{i}-r^{2} e_{i}$. So $\left(r e_{i}\right) u \neq r^{2} e_{i}$, contradiction. Similarly $u^{2} r=r^{3}=$ $u r^{2}$. Then $r \leqslant u$ and so $r=\sup _{i} r e_{i}$, as desired. 
(ii) Clearly $\alpha$ is a homomorphism, so it suffices to show that $\alpha$ is one-to-one. But $\alpha(x)=\alpha(y)$ implies $\left(x e_{i}\right)_{i \in I}=\left(y e_{i}\right)_{i \in I}$. From (i) it follows that $x=\sup _{i}\left(x e_{i}\right)=$ $\sup _{i}\left(y e_{i}\right)=y$. Hence $\alpha$ is a monomorphism.

Definition. A subset $S$ of $R$ is called orthogonal if $x^{n} y^{m}=0$ for every two distinct elements $x, y$ of $S$ and for every natural numbers $n, m$.

DEFINITION. $R$ is called orthogonally complete if $\sup S$ exists for every orthogonal subset $S$ of $R$.

REMARK. It is clear that $R$ an associative ring and $S$ an orthogonal set of $R$ implies $S^{+}$is an orthogonal set of $R^{+}$. Hence $R$ orthogonally complete implies $R^{+}$ orthogonally complete.

LEMMA 5. Let $\left(r_{i}\right)_{i \in I}$ be a subset of $R$ such that $\sup _{i} r_{i}$ exists, and let $e$ be $a$ hyperatom. Then $\sup _{i} e r_{i}$ exists, and furthermore $\sup _{i} e r_{i}=e \sup _{i} r_{i}$.

Proof. Let $z=\sup _{i} r_{i}$. As $r_{i} \leqslant z$ for every $i$, we have

$$
r_{i} z=r_{i}^{2}
$$

and

$$
r_{i}^{2} z=r_{i} z^{2}=r_{i}^{3}
$$

Thus,

$$
\begin{aligned}
& \left(e r_{i}\right)(e z)=e\left(r_{i} z\right) \stackrel{*}{=} e r_{i}^{2}=\left(e r_{i}\right)^{2} \\
& \left(e r_{i}\right)^{2}(e z)=\left(e r_{i}^{2}\right)(e z)=e\left(r_{i}^{2} z\right) \stackrel{* *}{=} e r_{i}^{3}=\left(e r_{i}\right)^{3} \\
& \left(e r_{i}\right)(e z)^{2}=\left(e r_{i}\right)\left(e z^{2}\right)=e\left(r_{i} z^{2}\right) \stackrel{* *}{=} e r_{i}^{3}=\left(e r_{i}\right)^{3}
\end{aligned}
$$

So, $e r_{i} \leqslant e z$ for every $i$. Thus, $e z$ is an upper bound of the set $\left(e r_{i}\right)_{i \in I}$. Let $u$ be any upper bound of $\left(e r_{i}\right)_{i \in I}$ Then $\left(e r_{i}\right) u=\left(e r_{i}\right)^{2}=e r_{i}^{2}$ and $\left(e r_{i}\right)^{2} u=\left(e r_{i}^{2}\right) u=\left(e r_{i}\right) u^{2}$ $=\left(e r_{i}\right)^{3}=e r_{i}^{3}$. Thus,

$$
r_{i}^{2}=r_{i}^{2}+u\left(e r_{i}\right)-e r_{i}^{2}=r_{i}(z+u e-e z)
$$

and

$$
r_{i}^{3}=r_{i}^{3}+u\left(e r_{i}^{2}\right)-e r_{i}^{3}=r_{i}^{2}(z+u e-e z)
$$

So

$$
\begin{aligned}
r_{i}(z+u e-e z)^{2} & =r_{i}\left(z^{2}+(u e)^{2}+(e z)^{2}+2 u e z-2 e z^{2}-2 u e(e z)\right) \\
& =r_{i}\left(z^{2}+u^{2} e+e z^{2}+2 u e z-2 e z^{2}-2 u e z\right)=r_{i}\left(z^{2}+u^{2} e-e z^{2}\right) \\
& =z^{2} r_{i}+r_{i}\left(u^{2} e\right)-\left(e r_{i}\right) z^{2}=r_{i}^{3}+e r_{i}^{3}-e r_{i}^{3}=r_{i}^{3} .
\end{aligned}
$$

Therefore $r_{i} \leqslant z+u e-e z$ for every $i$, and so, $z \leqslant z+u e-e z$ which implies that $z^{2}=z(z+u e-e z)$. Then $(u e) z=e z^{2}=(e z)^{2}$.

Also $z^{2}(z+u e-e z)=z^{3}$ implies $(u e) z^{2}=e z^{3}=(e z)^{3}$. Furthermore, $z^{3}=$ $z(z+u e-e z)^{2}=z\left(z^{2}+u^{2} e-e z^{2}\right)$ implies $\left(u^{2} e\right) z=u^{2}(e z)=e z^{3}=(e z)^{3}$. Consequently, it follows that $e z=u$ and so $e z=\sup _{i} e r_{i}$, as desired. 
THEOREM 3. Let $R$ be a Jordan ring with $\leqslant$ an order relation on $R$ such that $R$ is hyperatomic and orthogonally complete. Then $R$ is isomorphic to a direct product of Jordan division rings.

Proof. In view of Lemma 4(ii), it will suffice to show that the mapping $\alpha$ is an onto mapping. Let $\left(x_{i} e_{i}\right)_{i \in I} \in \prod_{i} R e_{i}$. For $n, m \in N$, we have

$$
\begin{aligned}
\left(x_{i} e_{i}\right)^{n}\left(x_{j} e_{j}\right)^{m} & =\left(x_{i}^{n} e_{i}\right)\left(x_{j}^{m} e_{j}\right)=e_{j}\left(x_{i}^{n} e_{i} x_{j}^{m}\right) \\
& =\left(\left(x_{i}^{n} x_{j}^{m}\right) e_{i}\right) e_{j}=\left(x_{i}^{n} x_{j}^{m}\right)\left(e_{i} e_{j}\right)=0
\end{aligned}
$$

and so $\left(x_{i} e_{i}\right)_{i \in I}$ is an orthogonal subset of $R$. As $R$ is orthogonally complete, $h=\sup _{i} x_{i} e_{i}$ exists. But then, from Lemma 5, it follows that for every element $j$ of $I$,

$$
h e_{j}=e_{j} \sup _{i}\left(x_{i} e_{i}\right)=\sup _{i}\left(\left(x_{i} e_{i}\right) e_{j}\right)=x_{j} e_{j}
$$

Hence $\alpha(h)=\left(h e_{i}\right)_{i \in I}=\left(x_{i} e_{i}\right)_{i \in I}$ so that $\alpha$ is an onto mapping.

THEOREM 4. Let $R$ be a Jordan ring that is a direct product of Jordan division rings. Then, the relation $\leqslant$ is a partial order on $R$ and $R$ is hyperatomic and orthogonally complete.

Proof. If $F$ is a Jordan division ring and $x \leqslant y$ we have $y U_{x}=x^{3}=x U_{x}$ and so $(y-x) U_{x}=0$. But $x \neq 0$ implies $U_{x}$ invertible on $F$ and so $y-x=0$, that is $y=x$. Hence $x \leqslant y$ if and only if $x=0$ or $x=y$. Then $\leqslant$ is a partial order on $F$.

Now, we consider the direct product $\prod_{i \in I} F_{i}$ and let $x=\left(x_{i}\right)_{i \in I}, y=\left(y_{i}\right)_{i \in I}$ be two elements in $\prod_{i \in I} F_{i}$. It is clear that $x \leqslant y$ if and only if $x_{i} \leqslant y_{i}$ for every $i$. So $\leqslant$ is a partial order on $R=\prod_{i \in I} F_{i}$.

On the other hand, from Remark 2, the unit element $e_{i}$ of $F_{i}$ is the only hyperatom of $F_{i}$, and it is clear that the hyperatoms of $R=\Pi F_{i}$ are the elements $\left(\alpha_{j}\right)$ such that there exists $i$ with $\alpha_{i}=e_{i}$ and $\alpha_{j}=0$ for every $j \neq i$.

If $x=\left(x_{i}\right)_{i \in I} \neq 0$, there exist $i$ such that $x_{i} \neq 0$. Thus $e x \neq 0$ with $e=\left(\alpha_{i}\right)$ the hyperatom such that $\alpha_{i}=e_{i}, \alpha_{j}=0$ if $i \neq j$. (Obviously $e$ is not the only hyperatom such that $e x \neq 0$. Any hyperatom $e^{\prime}=\left(\beta_{i}\right)$ of $R$ verifies this condition if $\beta_{i}=e_{i}$ for some $i$ such that $x_{i} \neq 0$.)

It is clear that $F_{i}$ is orthogonally complete for every $i$. Next, let $X=\left\{x^{(n)}\right\}_{n \in N}$ be an orthogonal subset of $\Pi F_{i}$. Then there exists at most one $n_{i}$ such that $x_{i}^{\left(n_{i}\right)} \neq 0$, for each $i$. If $x=\left(\beta_{i}\right)$ with $\beta_{i}=x_{i}^{\left(n_{i}\right)}$ if $x_{i}^{\left(n_{i}\right)} \neq 0$ and $\beta_{j}=0$ if there is no $m$ such that $x_{j}^{(m)} \neq 0$, it is clear that $x$ is the supremum of $X$. Hence $\Pi F_{i}$ is orthogonally complete and the theorem has been proved.

With Theorems 3 and 4 we have the following structure theorem

THEOREM. Any Jordan ring $R$ is isomorphic to a direct product of Jordan division rings if and only if the relation $\leqslant$ (defined by $x \leqslant y$ if $x y=x^{2}$ and $x^{2} y=x y^{2}=x^{3}$ ) is a partial order on $R$ such that $R$ is hyperatomic and orthogonally complete. 
ACKNOWLEDGMENTS. The authors would like to thank the referee for his valuable comments and suggestions.

\section{REFERENCES}

1. A. Abian, Direct product decomposition of commutative semisimple rings, Proc. Amer. Math. Soc. 24 (1970), 502-507.

2. , Order in a special class of rings and a structure theorem, Proc. Amer. Math. Soc. 52 (1975), 45-49.

3. Soc. 61 (1976), 188.

4. M. Chacron, Direct product of division rings and a paper of Abian, Proc. Amer. Math. Soc. 29 (1971), 259-262.

5. H. C. Myung and L. R. Jimenez, Direct product decomposition of alternative rings, Proc. Amer. Math. Soc. 47 (1975), 53-60.

6. K. A. Zhevlakov, A. M. Slinko, I. P. Shestakov, and A. I. Shirshov, Rings that are nearly associative, Academic Press, 1982.

Department of Algebra, University of Zaragoza, Zaragoza, Spain 